\title{
Diet-related changes of basal lamina fenestrations in the villous epithelium of the rat small intestine: Statistical analysis on scanning electron microscopy
}

\author{
Masatoshi Sato ${ }^{1,2}$, Keisuke Morita ${ }^{1}$, Rie Azumi ${ }^{1}$, Yusuke Mizutani ${ }^{2,3}$, Manabu Hayatsu ${ }^{2}$, Tatsuo Ushiki ${ }^{2}$, and \\ Shuji TeraI ${ }^{1}$ \\ ${ }^{1}$ Division of Gastroenterology and Hepatology, ${ }^{2}$ Division of Microscopic Anatomy, Niigata University Graduate School of Medical and \\ Dental Sciences, 1-757 Asahimachidori, Chuo-ku, Niigata, Japan and ${ }^{3}$ Office of Institutional Research, Hokkaido University, Kita 8 \\ Nishi 5, Kita-ku, Sapporo, Hokkaido, Japan
}

(Received 20 November 2021; and accepted 21 December 2021)

\begin{abstract}
The epithelial basal lamina of the small intestine has numerous fenestrations for intraepithelial migration of leukocytes. We have reported dynamic changes of fenestrations in dietary conditions. To investigate this phenomenon, we performed statistical analyses using scanning electron microscopy images of the epithelial basal lamina of rat intestinal villi after removal of the villous epithelium by osmium maceration. We examined structural changes in the number and size of fenestrations in the rat jejunum and ileum under fasted and fed states for $24 \mathrm{~h}$. Our findings revealed that, in the jejunum, the number of free cells migrating into the epithelium through fenestrations increased from $2 \mathrm{~h}$ after feeding, resulting in an increase in the fenestration size of intestinal villi; the number of free cells then tended to decrease at $6 \mathrm{~h}$ after feeding, and the fenestration size also gradually decreased. By contrast, the increase in the fenestration size by feeding was not statistically significant in the ileum. These findings indicate that the number of migrating cells increases in the upper part of the small intestine under dietary conditions, which may influence the absorption efficiency of nutrients including lipids, as well as the induction of nutrient-induced inflammation.
\end{abstract}

\section{INTRODUCTION}

The intestinal absorptive epithelium is a cellular layer covering small intestinal villi; it acts as a mechanical barrier between luminal contents and the underlying tissue including immune cells while simultaneously transporting substances like water, ions, and nutrients (Kiela and Ghishan 2016; Chelakkot et al. 2018). The basal lamina of this epithelium limits the contact between epithelial cells and the

Address correspondence to: Masatoshi Sato and Shunji Terai, Division of Gastroenterology and Hepatology, Niigata University Graduate School of Medical and Dental Sciences, 1-757 Asahimachidori, Chuo-ku, Niigata, Japan

Tel: +81-25-227-2207, Fax: +81-25-227-0776

E-mail:masatosa@med.niigata-u.ac.jp; terais@med. niigata-u.ac.jp underlying tissue.

Among the various nutrients, carbohydrates and proteins are absorbed from the luminal membrane of epithelial cells as monosaccharides and amino acids and released into the intercellular space of the epithelium, which results in their accumulation in the blood capillaries of the lamina propria. Neutral fats are also absorbed into the epithelial cells as monoglycerides and fatty acids, resynthesized into triacylglycerol in the cells, released into the intercellular space of the epithelium, and finally accumulate in the initial lymphatics of the lamina propria (Kiela and Ghishan 2016). The basal lamina is thus considered a filtration barrier for these nutrients as they need to pass from the epithelium to the lamina propria (Mantaj et al. 2018).

On the other hand, some studies pointed out that chylomicron particles are too large (ca. $100 \mathrm{~nm}$ to $1 \mu \mathrm{m}$ in diameter) to pass through the basal lamina 
without interruptions and suggested that the breaks in the basal lamina may facilitate the movement of chylomicrons from the intercellular space to the lamina propria (Tso and Balint 1986).

Previous studies using scanning electron microscopy (SEM) have revealed that the epithelial basal lamina of the small intestinal villus is a continuous layer but has numerous fenestrations, which are probably produced by the intraepithelial migration of free cells such as lymphocytes, eosinophils, and macrophages (McClugage and Low 1984; Komuro 1985; Takahashi-Iwanaga H et al. 1999; Takeuchi and Gonda 2004).

We recently introduced a reliable technique for directly visualizing the intestinal basal lamina after removal of the the villous epithelium and clarified a unique phenomenon that the size of basal lamina fenestrations increases in the rat jejunum after feeding (Azumi et al. 2018). We also suggested that basal lamina fenestrations are changed according to the dynamics of migrating leukocytes under certain dietary conditions.

To further clarify the role of fenestrations in lipid absorption, we investigated the dynamics of basal lamina fenestrations under high-fat diet condition (Morita et al. 2019). We showed that basal lamina fenestrations provide important pathways for the release of chylomicrons from the epithelial side to the side of the lamina propria and proposed that dynamic changes in basal lamina fenestration may play an important role in increasing or decreasing the efficiency of lipid absorption (Morita et al. 2019).

These previous studies focused on short-term effects (i.e., $2 \mathrm{~h}$ after feeding) on the intestinal epithelium. Thus, the time course and dynamics in basal lamina fenestrations are still not fully elucidated yet. This study aimed to further strengthen our previous findings and establish the morphological basis of the dynamics of intestinal basal lamina fenestrations in response to dietary conditions. Using SEM, we observed fenestrations in the rat basal lamina of intestinal villi after removal of the villous epithelium, and statistically analyzed structural changes in fenestrations of the jejunum and ileum under fasted and fed states for $24 \mathrm{~h}$ (i.e., $0,2,4,6,12$ and $24 \mathrm{~h}$ after feeding).

In this study, we showed that free cells migrating through fenestrations actually increase from $2 \mathrm{~h}$ after feeding, which results in an increase in the fenestration size (area) in each villus, especially in the jejunum. These findings are discussed, especially in relation to the possible regulation of nutrient absorption.

\section{MATERIALS AND METHODS}

Animals and experimental design (Fig. 1). Six-weekold male Wistar rats were purchased from Japan SLC, Inc. (Shizuoka, Japan). The animals were divided into two groups: 9 animals were used for the fasting group, while 18 animals were included in the feeding group. All animals were fasted (with free access to water) $20 \mathrm{~h}$ before experimentation. The animals in the fasting group then extended fasting for 0,2 , or $4 \mathrm{~h}$ ( 3 animals per condition), while the animals in the feeding group were fed pellets (Rodent Lab Diet 5L37; PMI Nutrition International Inc., Brentwood, MO, USA) for 0, 2, 4, 6, 12, or $24 \mathrm{~h}$ (3 animals per condition). The rats were anesthetized by intraperitoneal injection of pentobarbital sodium and then transcardially perfused with physiological saline followed by $4 \%$ paraformaldehyde in $0.1 \mathrm{M}$ phosphate buffer. This study was performed in accordance with the Niigata University Animal Experimental Guidelines after approval by the Ethics Committee for Niigata University Animal Experimentation (No. 340-2).

SEM preparation. In this study, the segment of upper two-fifths of the small intestine was defined as jejunum, while the remaining lower segment was referred to ileum. After perfusion fixation, the middle of the jejunum and the middle of ileum were removed from the body, sliced to a thickness of approximately $0.3 \mathrm{~mm}$ perpendicular to the long axis of the intestine, and fixed again with $2 \%$ glutaraldehyde for $48 \mathrm{~h}$. Subsequently, to remove the epithelium, osmium maceration plus ultrasonic treatment was performed according to the method of Azumi et al. (2018) (Takeuchi and Gonda 2004). Briefly, the slices were immersed in a $2 \%$ aqueous solution of osmium tetroxide at $20^{\circ} \mathrm{C}$ for $72 \mathrm{~h}$, followed by treatment of the slices in an ultrasonic bath (Bransonic Ultrasonic Cleaner; Branson Ultrasonics, Enerson Japan, Atsugi, Japan) until the epithelium was completely removed from the intestinal villi.

Conductive staining was then performed on a $1 \%$ tannic acid aqueous solution for $2 \mathrm{~h}$ followed by a $1 \%$ osmium tetroxide aqueous solution for $2 \mathrm{~h}$. These slices were dehydrated in a graded series of ethanol, dried using a critical point dryer with $\mathrm{CO}_{2}$, mounted on aluminum stubs, and metal-coated with platinum-palladium for SEM observation.

SEM imaging and statistical imaging analysis. The basal lamina of the small intestinal villi was observed using a Scanning Electron Microscope (S-3700N; 


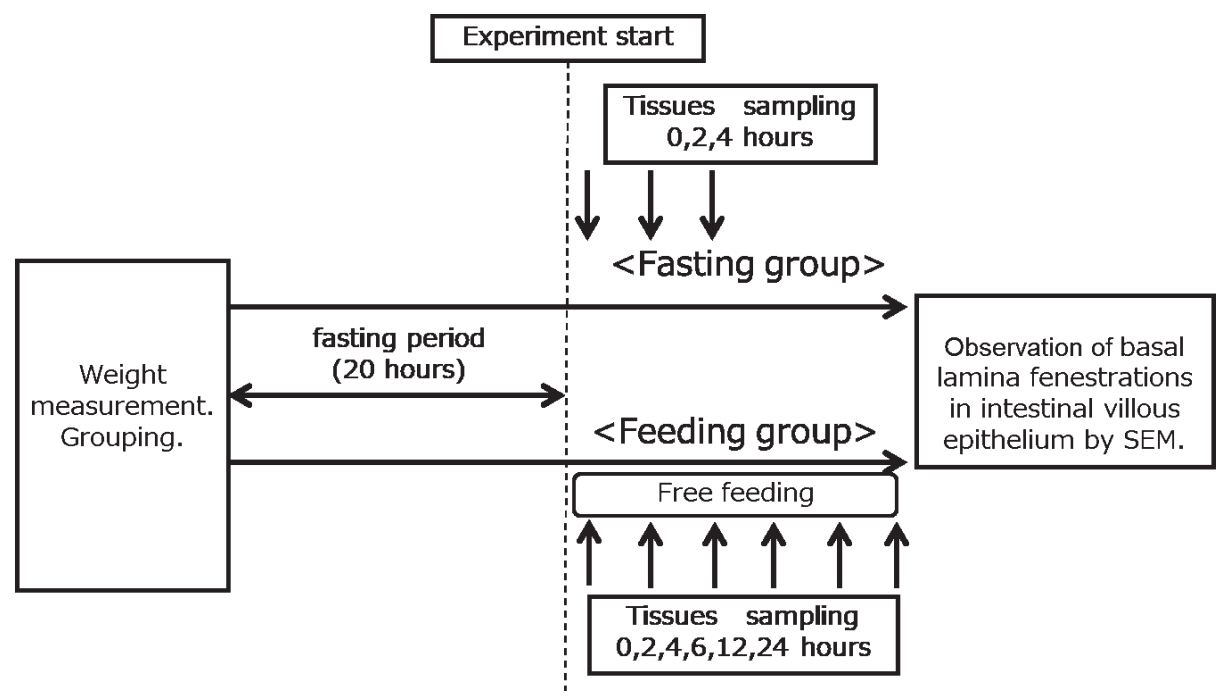

Fig. 1 Experimental design. Rats, all of which were fasted for $20 \mathrm{~h}$, were divided into two experimental groups: a fasting group and a feeding group. In the fasting group, the animals were further fasted and killed at 0,2 , and $4 \mathrm{~h}$ after the experiment start. In the feeding group, the animals were fed solid pellets, and killed after $0,2,4,6,12$, and $24 \mathrm{~h}$. The basal lamina fenestrations in the intestinal villous epithelium were then exposed to osmium maceration plus ultrasonic treatment and observed using scanning electron microscopy (SEM).

Hitachi) at an acceleration voltage of $10 \mathrm{kV}$. The working distance for imaging was set at $10 \mathrm{~mm}$, and both secondary electron (SE) and backscattered electron (BSE) images of single foliated villi were carefully captured at an image magnification of $250 \times$ after the flat surface was positioned horizontally with respect to the imaging surface.

We selected images of 30 different villi from each group for statistical analysis. Calculations were made in the middle $60 \%$ of the entire height of each villus to avoid preparation artifacts such as wrinkles that were often present at the villous top and base (Fig. 2). Using the BSE images, the number and area of fenestrations, as well as the area of the epithelial basal lamina, were measured for each villus using the ImageJ software (National Institutes of Health, Bethesda, MD, USA; http://imagej.nih.gov/ $\mathrm{ij} /$ ). As a first step, a binary image of each micrograph was created from the original BSE image using ImageJ. Because fenestrated areas appear in BSE images dark against the bright surface of the basal lamina, almost all fenestrated areas could be extracted from these binary images. We carefully compared the binary images with the original images and manually selected fenestrations with and without attached free cells from these images. Because each villus has a different shape and size, we calculated 1) the average number of fenestrations per unit area, 2) the average area of fenestrations per unit area, and 3) the average number of free cells relative to the number of fenestrations in these images.

In the fasting group, we compared the changes in the above three parameters at each time point. In the feeding group, we compared the fenestration changes of the above three parameters between the pre-feeding point $(0 \mathrm{~h})$ and each time point after feeding.

The Kruskal-Wallis test (Kruskal and Wallis 1952) was performed using the statistical analysis software SPSS (IBM SPSS Statistics Subscription; IBM, Armonk, NY, USA) for the comparison of body weights between the fasting and feeding groups, for the comparison of fenestration changes in the fasting group, and for the comparison of fenestration changes between pre-feeding point $(0 \mathrm{~h})$ and each time point after feeding in the feeding group. Additionally, the Dunn test was performed with a significance level of 0.05 , and the Bonferroni correction was applied to the $P$-value for comparisons between two different time points in the fasting group, as well as for comparisons in the feeding group between the pre-feeding time point $(0 \mathrm{~h})$ and each time point after feeding. In all statistical tests, a $P$-value $<0.05$ was considered statistically significant.

\section{RESULTS}

In this study, the average body weight was not significantly different between the fasting and feeding groups $(P=0.957$; Table 1$)$. Osmium maceration 
plus ultrasonic treatment was effective in removing the villous epithelial cells from the specimens. Thus, the entire surface of the villous basal lamina was clearly observed by SEM (Fig. 2). BSE images were especially useful for studying the shape and size of the fenestrations in the villous basal lamina because the observed fenestrations were dark enough to be clearly detectable for further statistical analyses.

Table 1 Body weight of each group

\begin{tabular}{cc}
\hline Group & Average weight $(\mathrm{g}) \pm$ Standard error \\
\hline Fasting group 0 h & $132.9 \pm 3.4$ \\
Fasting group 2 h & $134.3 \pm 2.9$ \\
Fasting group 4 h & $133.2 \pm 0.8$ \\
Feeding group 0 h & $134.0 \pm 0.5$ \\
Feeding group 2 $\mathrm{h}$ & $133.1 \pm 2.2$ \\
Feeding group 4 h & $133.7 \pm 1.7$ \\
Feeding group 6 h & $131.7 \pm 1.8$ \\
Feeding group 12 h & $134.2 \pm 1.1$ \\
Feeding group 24 h & $132.5 \pm 2.0$ \\
\hline
\end{tabular}

The weight did not significantly differ between the two groups at corresponding time points.
Changes in number and size of fenestrations in the villous epithelial basal lamina

SEM images of small intestinal villi showed no apparent changes in the number and size of fenestrations at each sampling time in the fasting group in both the jejunum and the ileum. By contrast, the fenestrations appeared to widen from $2 \mathrm{~h}$ after feeding, especially in the jejunum (Fig. 3). To quantify these changes in both groups, statistical analyses were performed.

Jejunum: The quantitative analysis in the fasting group showed no significant differences in the fenestration number per villus area $(P=0.50)$ or in the fenestration area per villus area $(P=0.40)$ among the different sampling points (Tables 2 and 3 ). In the feeding group, there was no change in the fenestration number per villus area during the time course after feeding, similar to the observation in the fasting group $(P>0.05$; Table 4$)$. However, the total fenestration area per villus area significantly increased $2 \mathrm{~h}$ after feeding in comparison to the control fasting group $(P<0.05$; Table 5 and Fig. 4$)$, and decreased again $4 \mathrm{~h}$ or later. The differences in the total fenestration area per villus area were not statistically significant between the control fasting group and $4-24 \mathrm{~h}$ after feeding groups $(0 \mathrm{~h} ; P>0.05)$, al-
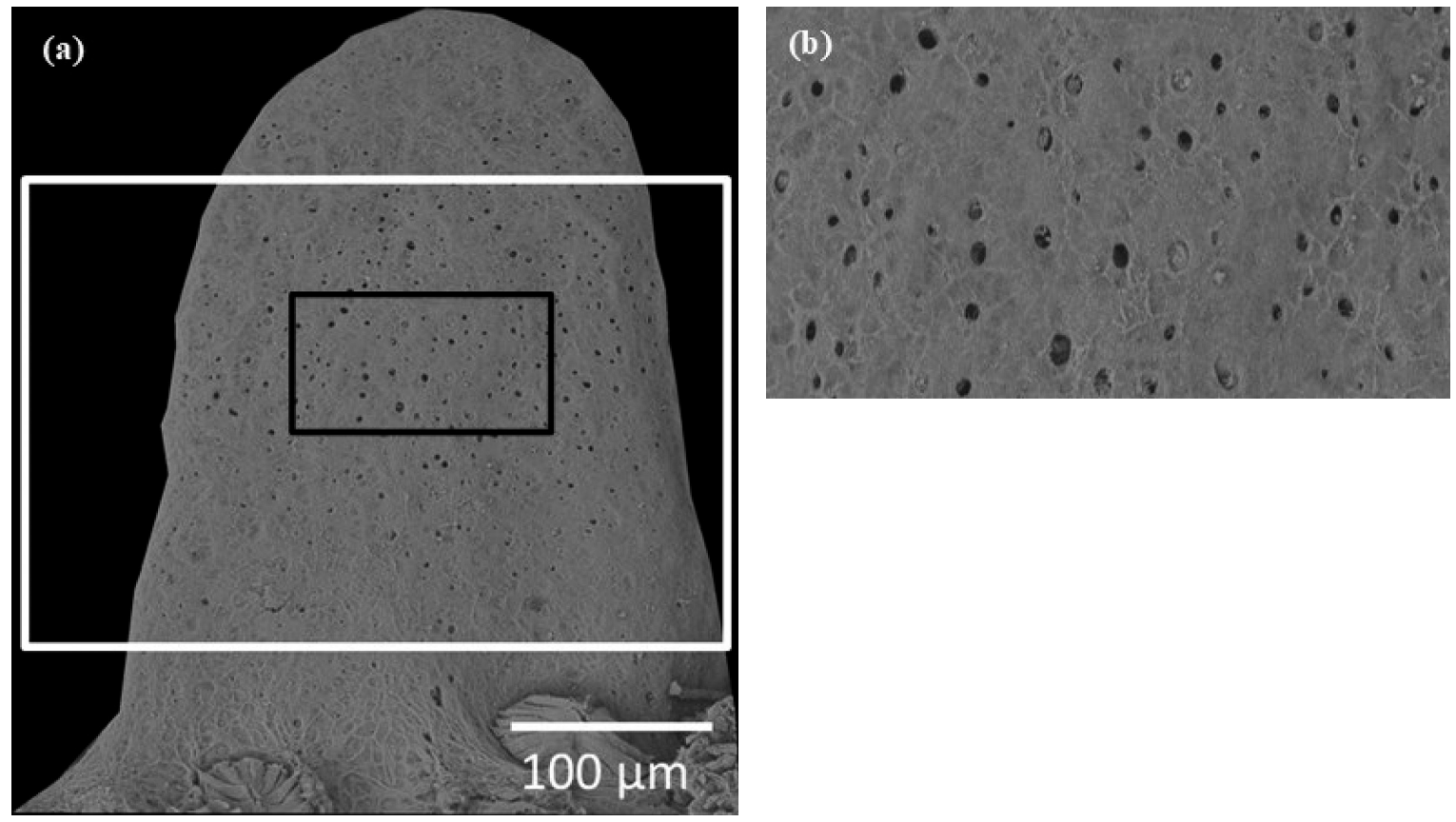

Fig. 2 BSE image of an intestinal villus in the rat jejunum after osmium maceration plus ultrasonic treatment. The villous epithelial cells are completely removed from the villus, and the entire surface of the villous basal lamina is exposed in this image (a). A high magnification image of the black square in (a) clearly shows fenestrations with various size and free cells passing through fenestrations (b). The white square in (a) shows the analysis area: the middle 60\% of the entire height of the villus was selected to avoid preparation artifacts such as wrinkles often present at the villus top and base. 

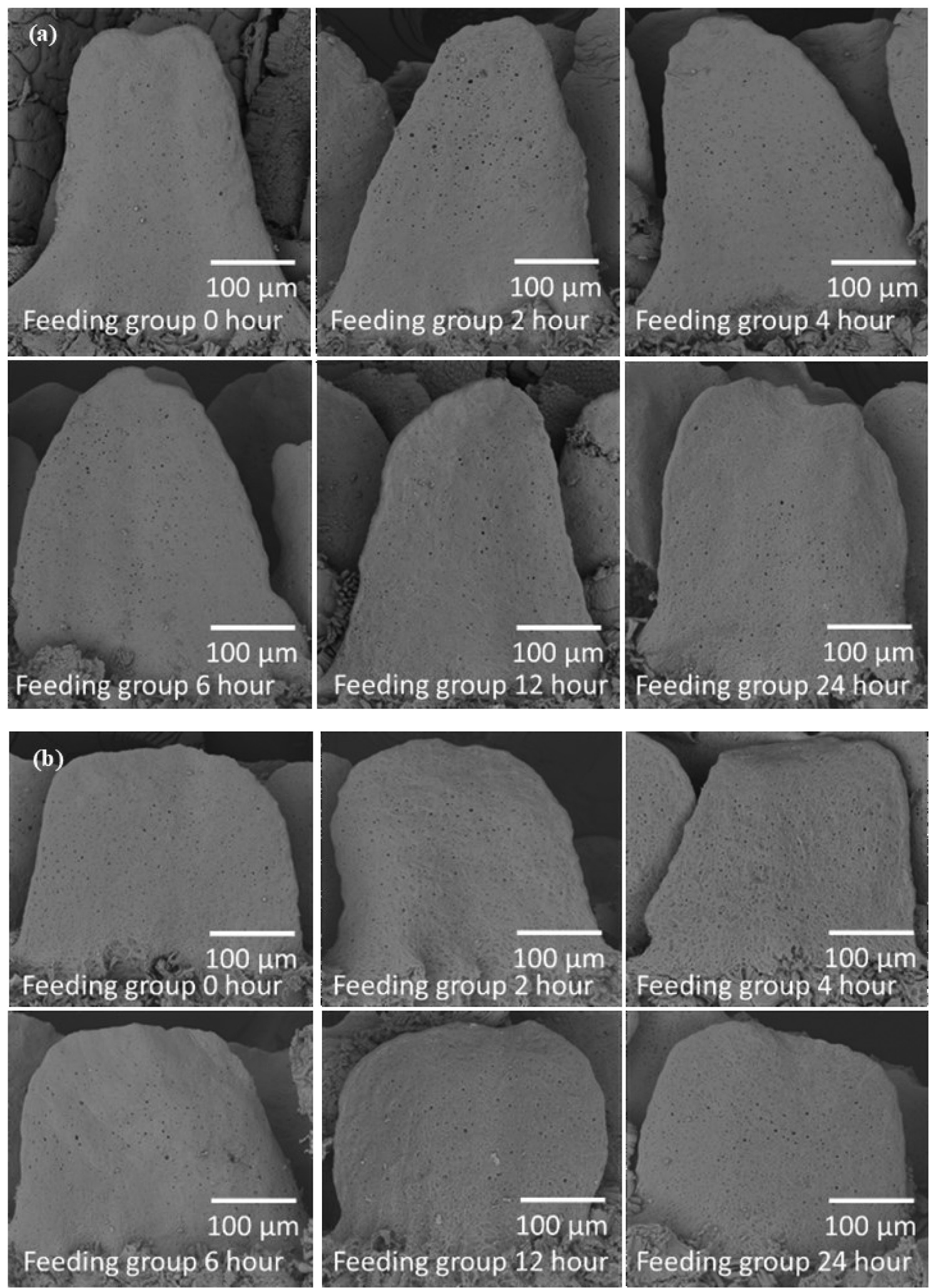

Fig. 3 BSE images depicting the basal lamina of intestinal villi in the jejunum (a) and ileum (b) of the feeding group. Each image shows a typical scanning electron microscopy image at each sampling point. These images suggest that the fenestration size increases from $2 \mathrm{~h}$ after feeding, especially in the jejunum. 
Table 2 Number of fenestrations per villus area in the fasting group (jejunum)

\begin{tabular}{cc}
\hline Group & $\begin{array}{c}\text { Number of fenestrations/villus area } \times \\
10^{2}\left(/ \mu \mathrm{m}^{2}\right) \pm \text { Standard error }\end{array}$ \\
\hline Fasting group 0 $\mathrm{h}$ & $10.824 \pm 0.370$ \\
Fasting group 2 $\mathrm{h}$ & $11.941 \pm 0.514$ \\
Fasting group 4 h & $11.400 \pm 0.503$ \\
\hline
\end{tabular}

No significant difference was found between the sampling points in the jejunum.

Table 4 Number of fenestrations per villus area in the feeding group (jejunum)

\begin{tabular}{cc}
\hline Group & $\begin{array}{c}\text { Number of fenestrations/villus area } \times \\
10^{2}\left(/ \mu \mathrm{m}^{2}\right) \pm \text { Standard error }\end{array}$ \\
\hline Feeding group 0 $\mathrm{h}$ & $11.855 \pm 0.484$ \\
Feeding group 2 $\mathrm{h}$ & $12.317 \pm 0.606$ \\
Feeding group 4 h & $10.947 \pm 0.487$ \\
Feeding group 6 h & $12.055 \pm 0.385$ \\
Feeding group 12 h & $12.024 \pm 0.503$ \\
Feeding group 24 h & $10.497 \pm 0.312$ \\
\hline
\end{tabular}

No significant difference was found between the sampling points in the jejunum $(P>0.05)$.

though the value was slightly greater in the latter groups.

Ileum: Similar to the jejunum, there was no significant difference in the number of fenestrations per villus area $(P=0.88$; Table 6$)$ or in the fenestration area per villus area $(P=0.11)$ at each sampling point in the fasting group. In the feeding group, there was also no significant change in the fenestration number per villus area or the fenestration area per villus area between the different sampling points after feeding $(P>0.05$; Table 6, Table 7, and Fig. 5).

Change in the number of migrating free cells

The number of free cells passing through the fenestrations of the villous epithelial basal lamina was studied in both fasting and feeding groups. SEM images suggested that the number of free cells adhering to the window increased in the feeding group compared to that in the fasting group (Fig. 6).

Jejunum: In the fasting group, the statistical analysis revealed no significant difference in the number of free cells relative to the number of fenestrations between the different sampling points $(P=0.06)$. However, in the feeding group, there was a significant increase in the mean number of free cells relative to the number of fenestrations at $2 \mathrm{~h}$ and $4 \mathrm{~h}$ after fasting $(P<0.05$; Table 8 and Fig. 7). This value then decreased after $6 \mathrm{~h}$ of feeding and gradually
Table 3 Percentage of the fenestration area to the villus area in the fasting group (jejunum)

\begin{tabular}{cc}
\hline Group & $\begin{array}{c}\text { Total fenestration area/villus area } \pm \\
\text { Standard error }\end{array}$ \\
\hline Fasting group 0 $\mathrm{h}$ & $1.883 \pm 0.050$ \\
Fasting group 2 $\mathrm{h}$ & $2.063 \pm 0.065$ \\
Fasting group 4 h & $1.944 \pm 0.101$ \\
\hline
\end{tabular}

No significant difference was found between the sampling points in the jejunum $(P=0.40)$.

Table 5 Percentage of the fenestration area to the villus area in the feeding group (jejunum)

\begin{tabular}{cc}
\hline Group & $\begin{array}{c}\text { Total fenestration area/villus area } \pm \\
\text { Standard error }\end{array}$ \\
\hline Feeding group 0 $\mathrm{h}$ & $1.878 \pm 0.043$ \\
Feeding group 2 $\mathrm{h}$ & $2.536 \pm 0.110(P<0.05)$ \\
Feeding group 4 h & $2.132 \pm 0.082$ \\
Feeding group 6 $\mathrm{h}$ & $2.124 \pm 0.054$ \\
Feeding group 12 h & $2.120 \pm 0.046$ \\
Feeding group 24 h & $2.090 \pm 0.054$ \\
\hline
\end{tabular}

The size of fenestrations in the jejunum significantly increased between $0 \mathrm{~h}$ and $2 \mathrm{~h}$ after feeding $(P<0.05)$. There was no statistical difference in the time points after $4 \mathrm{~h}$ of feeding compared to the time point before feeding $(0 \mathrm{~h} ; P \geq 0.05)$.

approached the level of the control fasting group by 24 of feeding.

Ileum: In the ileum, there was no significant difference in the number of free cells relative to the number of fenestrations in the fasting group $(P=0.15)$. In the feeding group, the number of free cells with respect to the fenestration number slightly increased at $2 \mathrm{~h}$ and $4 \mathrm{~h}$ after feeding, but no statistical significance was observed between different sampling points in the feeding group $(P=0.17$; Table 9 and Fig. 8).

\section{DISCUSSION}

The present study investigated the dynamic changes in the epithelial basal lamina fenestrations of rat intestinal villi in response to diet. Previously, we studied the structures of epithelial basal lamina fenestrations in rat intestinal villi by SEM and showed that the number and size of fenestrations increased $2 \mathrm{~h}$ after feeding in the jejunum (Azumi et al. 2018). In the present study, we further extended our studies on this phenomenon and examined fenestration changes over time for $24 \mathrm{~h}$ (i.e., 0, 2, 4, 6, 12, and $24 \mathrm{~h}$ ) after feeding both in the jejunum and ileum.

We clearly showed that the fenestration area per 

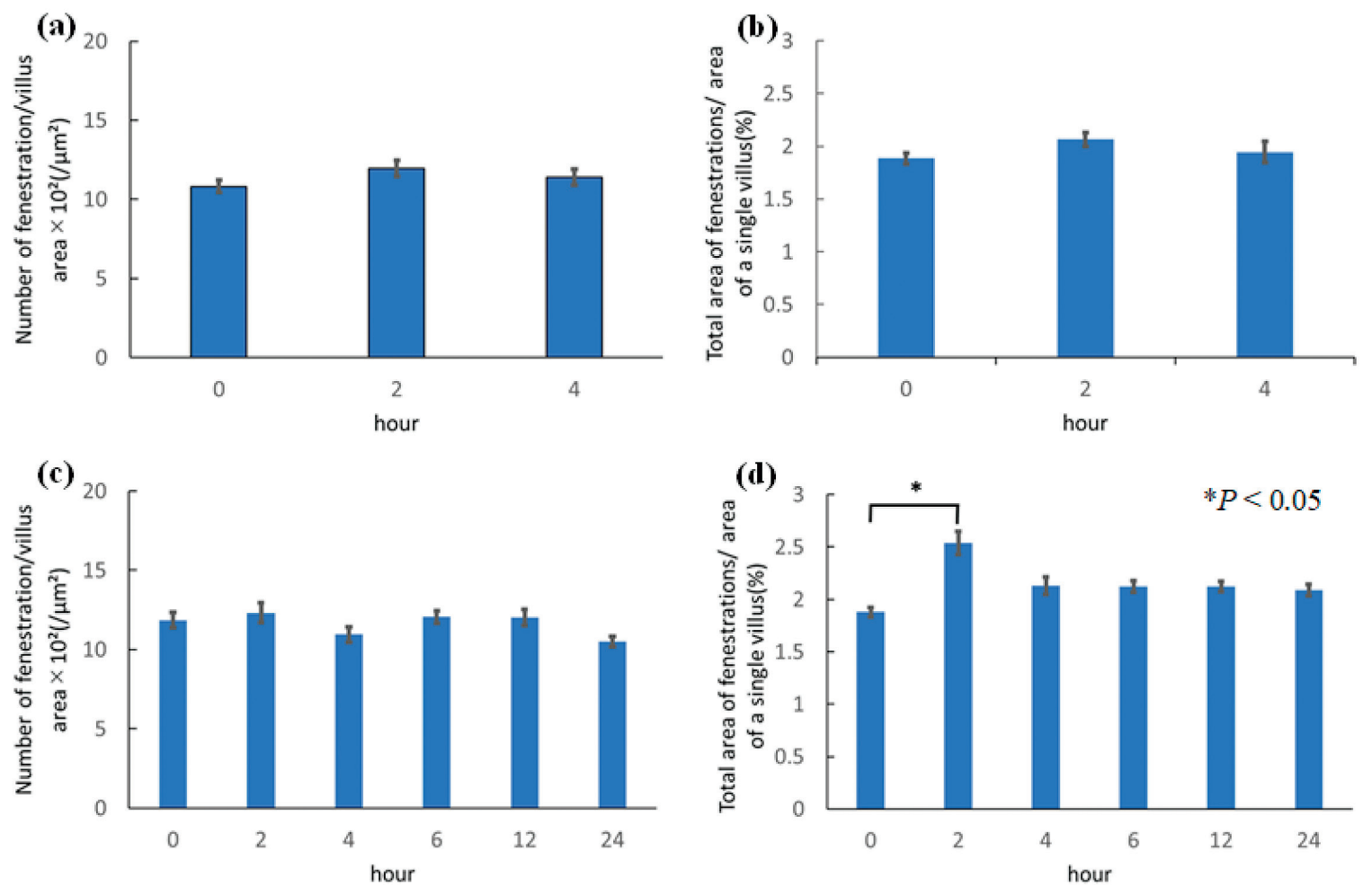

Fig. 4 Number and size of fenestration per unit area of the villus in the fasting group (a, b) and feeding group (c, d) in the jejunum. a. Fenestration number per villus area in the fasting group. b. Percentage of the fenestration area to villous area in the fasting group. c. Fenestration number per villus area in the feeding group. d. Percentage of the fenestration area to villous area in the feeding group. In the feeding group, the fenestration size significantly increases $2 \mathrm{~h}$ after feeding $\left({ }^{*} P<0.05\right)$.

Table 6 Number of fenestrations per villus area in the ileum

\begin{tabular}{cc}
\hline Group & $\begin{array}{c}\text { Number of fenestrations/villus area } \times \\
10^{2}\left(/ \mu \mathrm{m}^{2}\right) \pm \text { Standard error }\end{array}$ \\
\hline Fasting group 0 $\mathrm{h}$ & $11.029 \pm 0.368$ \\
Fasting group 2 $\mathrm{h}$ & $11.365 \pm 0.373$ \\
Fasting group 4 h & $11.568 \pm 0.650$ \\
Feeding group 0 $\mathrm{h}$ & $11.477 \pm 0.311$ \\
Feeding group 2 $\mathrm{h}$ & $11.107 \pm 0.318$ \\
Feeding group 4 h & $12.285 \pm 0.426$ \\
Feeding group 6 h & $10.471 \pm 0.499$ \\
Feeding group 12 $\mathrm{h}$ & $12.150 \pm 0.395$ \\
Feeding group 24 h & $12.405 \pm 0.378$ \\
\hline
\end{tabular}

No significant difference was found between the different sampling points in the fasting group $(P=0.88)$. In the feeding group, there was no significant change in the fenestration number per villus area between the different sampling points after feeding $(P \geq 0.05)$.

villus area indeed dramatically increased from $2 \mathrm{~h}$ after feeding in the jejunum, but not in ileum. On the other hand, our present study demonstrated that there was no change in the fenestration number per
Table 7 Percentage of the fenestration area to villous area in the ileum

\begin{tabular}{cc}
\hline Group & $\begin{array}{c}\text { Total fenestration area/villus area } \pm \\
\text { Standard error }\end{array}$ \\
\hline Fasting group 0 $\mathrm{h}$ & $2.171 \pm 0.054$ \\
Fasting group 2 h & $2.332 \pm 0.061$ \\
Fasting group 4 h & $1.955 \pm 0.118$ \\
Feeding group 0 $\mathrm{h}$ & $2.131 \pm 0.032$ \\
Feeding group 2 $\mathrm{h}$ & $2.296 \pm 0.040$ \\
Feeding group 4 $\mathrm{h}$ & $2.200 \pm 0.081$ \\
Feeding group 6 h & $1.985 \pm 0.090$ \\
Feeding group 12 h & $2.277 \pm 0.092$ \\
Feeding group 24 h & $2.309 \pm 0.043$ \\
\hline
\end{tabular}

No significant difference was found between the different sampling points in the fasting group $(P=0.11)$. In the feeding group, there was no significant change in the fenestration area per villus area between the different sampling points after feeding $(P \geq 0.05)$.

villus area for $24 \mathrm{~h}$ in both the jejunum and ileum in the feeding group, as well as in the fasting group. These findings indicated that the number of the fenestration is stable irrespective of fasting or feeding, 

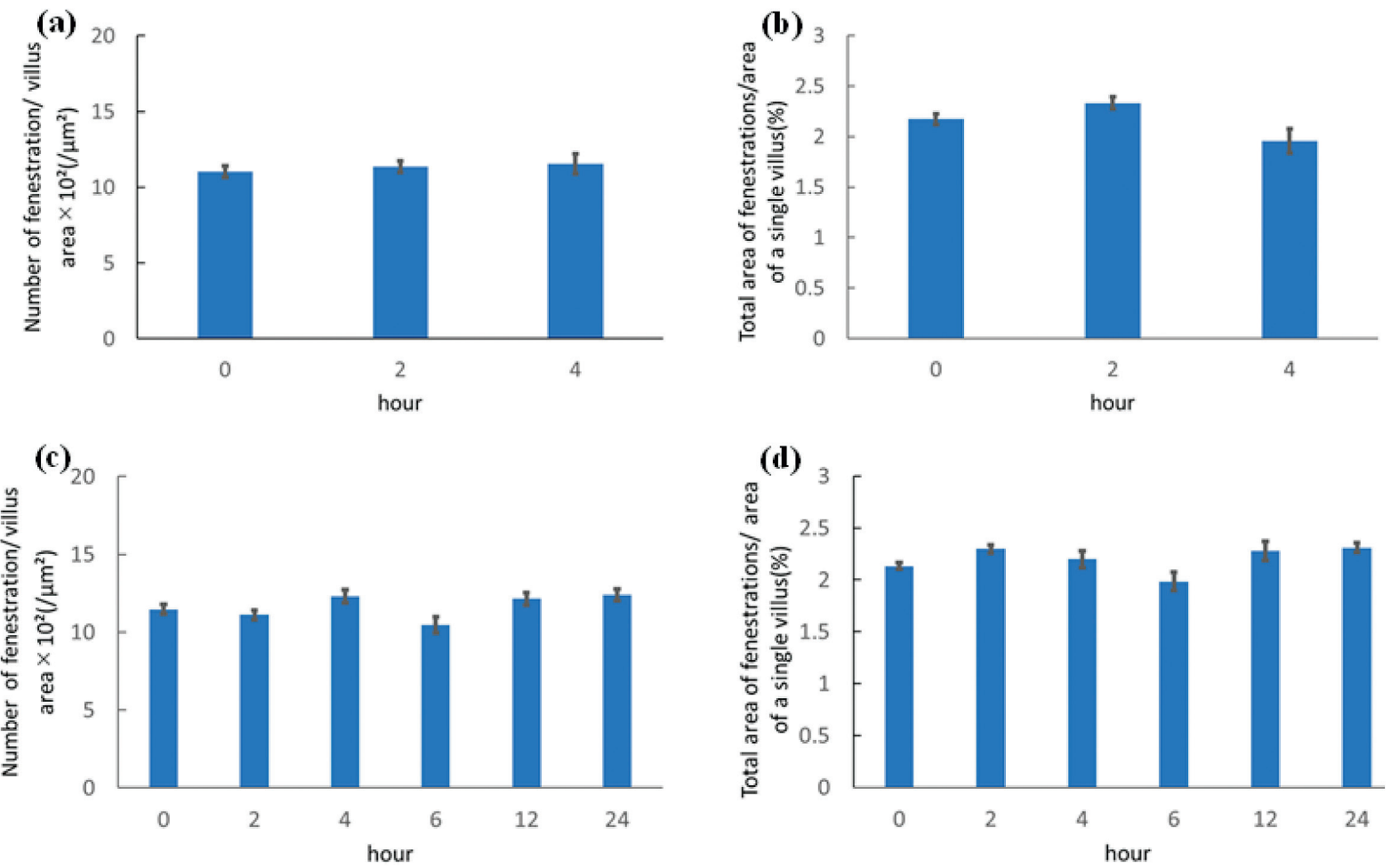

Fig. 5 Number and size of fenestrations in the fasting group (a. b) and feeding group (c, d) in the ileum. a. Fenestration number per villus area in the fasting group. b. Percentage of the fenestration area to villous area in the fasting group. c. Fenestration number per villus area in the feeding group. d. Percentage of the fenestration area to villous area in the feeding group.
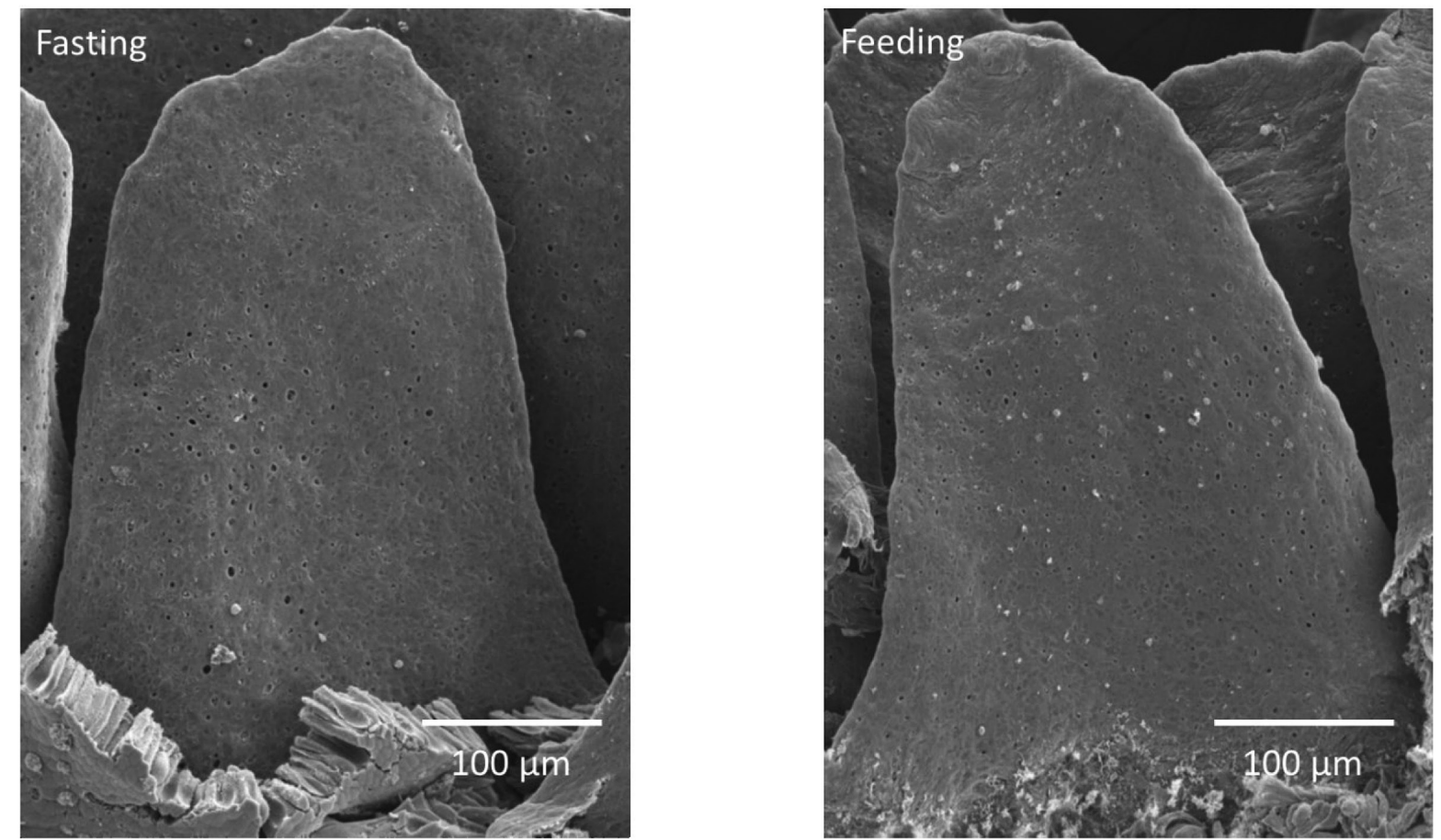

Fig. 6 Changes in the number of free cells attached to jejunal villi in the fasting group and the feeding group. The number of free cells is higher for the villus of the feeding group than that of the fasting group. 
Table 8 Percentage of the number of free cells to the total number of fenestrations (jejunum)

\begin{tabular}{cc}
\hline Group & $\begin{array}{c}\text { Number of free cells/number of total fenestrations } \pm \\
\text { Standard error }\end{array}$ \\
\hline Fasting group 0 $\mathrm{h}$ & $3.331 \pm 0.215$ \\
Fasting group 2 $\mathrm{h}$ & $3.075 \pm 0.301$ \\
Fasting group 4 h & $2.556 \pm 0.208$ \\
Feeding group 0 $\mathrm{h}$ & $2.709 \pm 0.247$ \\
Feeding group 2 $\mathrm{h}$ & $5.301 \pm 0.626(P<0.05)$ \\
Feeding group 4 h & $5.907 \pm 0.428(P<0.05)$ \\
Feeding group 6 h & $3.772 \pm 0.411$ \\
Feeding group 12 h & $3.143 \pm 0.281$ \\
Feeding group 24 h & $2.893 \pm 0.311$ \\
\hline
\end{tabular}

No significant difference in the number of free cells relative to the fenestration number was present between the different sampling points in the fasting group $(P=0.06)$. In the feeding group, there was a significant increase in the mean number of free cells relative to the fenestration number at $2 \mathrm{~h}$ and $4 \mathrm{~h}$ after fasting $(P<0.05)$.
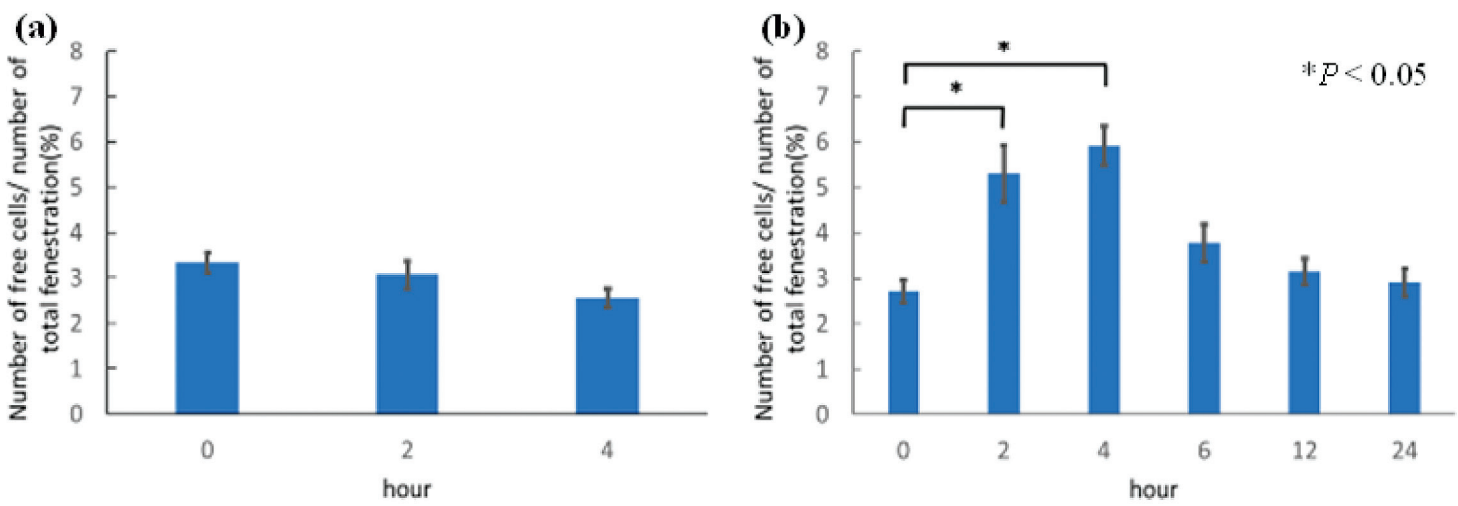

Fig. 7 Percentage of the number of free cells to the total number of fenestrations in the jejunum. a. Fasting group. b. Feeding group. The number of free cells is significantly increased in the feeding group at $2 \mathrm{~h}$ and $4 \mathrm{~h}\left({ }^{*} P<0.05\right)$.

but some of fenestrations dilate temporally at $2 \mathrm{~h}$ after feeding in the jejunum. We further demonstrated that the size of fenestrations decreased $6 \mathrm{~h}$ after feeding and gradually approached the level of the mean size in the jejunum of the fasting group. In addition, we showed that the number of free cells passing through the fenestrations increased in the jejunum $2 \mathrm{~h}$ and $4 \mathrm{~h}$ after feeding.

We previously suggested that under dietary conditions, basal lamina fenestrations are changeable through the dynamics of migrating leukocytes. Here, we confirmed the previous results and further added a new finding that the number of fenestrations in the villi was rather stable though the size of the fenestrations increased. This finding can be explained by the increase in free cells passing through the preexisting basal lamina fenestrations in response to feeding; it is probable that the majority of migrating cells pass through the preexisting fenestrations by pushing the small holes wide. Interestingly, some previous researchers stated that it takes more than $30 \mathrm{~min}$ for leukocytes passing through the intact vascular basal lamina and, if present, a tiny hole of the basal lamina could become a target site for migrating leukocytes penetrating the basal lamina (Walker et al. 1995; Hoshi and Ushiki 2004). The use of preexisting fenestrations may be smooth for rapid movement of migrating cells, and also be reasonable to maintain the barrier function of the basement membrane.

The present study further revealed, at first time, that the number of free cells decreased $6 \mathrm{~h}$ after feeding, which is consistent with the finding that the fenestration size gradually decreased $6 \mathrm{~h}$ after feeding. This phenomenon also suggests that the movement of free cells decreased with the passage of 
Table 9 Percentage of the number of free cells to the total number of fenestrations in the ileum

\begin{tabular}{cc}
\hline Group & $\begin{array}{c}\text { Number of free cells/number of total fenestrations } \pm \\
\text { Standard error }\end{array}$ \\
\hline Fasting group 0 h & $3.525 \pm 0.205$ \\
Fasting group 2 h & $3.088 \pm 0.301$ \\
Fasting group 4 h & $3.264 \pm 0.240$ \\
Feeding group 0 h & $3.029 \pm 0.225$ \\
Feeding group 2 h & $3.783 \pm 0.224$ \\
Feeding group 4 h & $4.573 \pm 0.362$ \\
Feeding group 6 h & $3.432 \pm 0.253$ \\
Feeding group 12 h & $3.457 \pm 0.268$ \\
Feeding group 24 h & $3.251 \pm 0.294$ \\
\hline
\end{tabular}

There was no significant difference in the number of free cells relative to the fenestration number in the fasting group $(P=0.15)$. In the feeding group, the number of free cells with respect to the fenestration number slightly increased at $2 \mathrm{~h}$ and $4 \mathrm{~h}$ after feeding, but no statistical significance was present between different sampling points in the feeding group $(P=0.17)$.
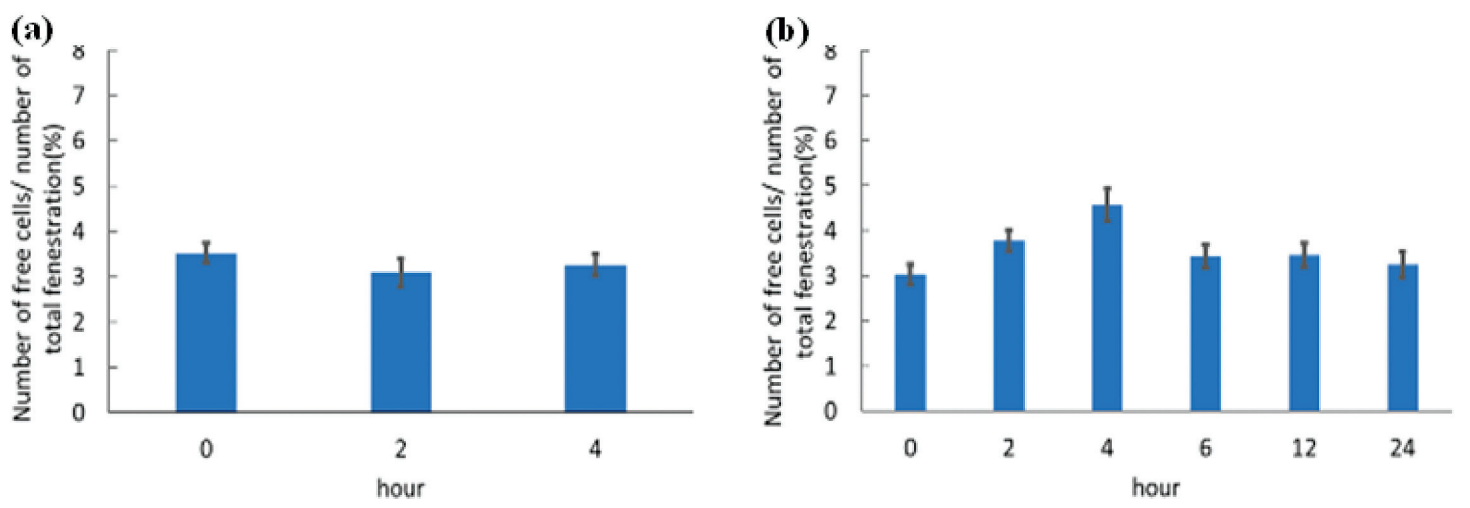

Fig. 8 Percentage of the number of free cells to the total number of fenestrations in the ileum. a. Fasting group. b. Feeding group.

time after feeding.

The mechanism by which the fenestrations closed over time after dilatation has not been clarified. One possible explanation is that collagen fibrils supporting the rim of each fenestration temporally bend outward by migrating cells but unbend after the event. Another possibility is the role of subepithelial fibroblasts underlying the basement membrane of villous epithelium. This subepithelial fibroblasts form a cellular network and are sensitive to mechanical stress; they cause a transient contraction of the network by elevation of intracellular $\mathrm{Ca}^{2+}$ and release of ATP from the cells (Furuya et al. 2005). Thus, the stimulation of subepithelial fibroblasts may be also concerned with the morphological changes of the fenestration.

The reason for the high migratory activity of leukocytes in the jejunum in response to feeding re- mains to be elucidated. One possibility is that free cells could increase in the villi of the small intestine as a part of the immune response to food. Previous studies have reported the presence of nutrient-induced inflammation in the intestine (Miura et al. 1998; Hara et al 2003; Ji et al. 2011). This inflammation is thought to be induced by the stimulation of cytokine release by intestinal immune cells and epithelial cells following fat absorption. Such inflammation might be explained by our previous findings that the number of free cells passing through fenestrations drastically increases in the upper jejunum $2 \mathrm{~h}$ after feeding a high-fat diet compared to those fed a normal diet (Morita et al. 2019).

It should be emphasized that the epithelial basal lamina forms a barrier between the intercellular space of the epithelium and the lamina propria and that the basal lamina fenestrations of the intestinal 
villi provide an important pathway for large nutrients, such as chylomicrons, to be released from the epithelial side to the space of the lamina propria (Azumi et al. 2018). Moreover, we also previously demonstrated that the number of free cells passing through the fenestrations increased in the upper jejunum after feeding a high-fat diet, and the size of the fenestrations in this region also increased (Morita et al. 2019). Because it is probable that the size and number of fenestrations are related to the efficiency of fat absorption, we strongly hypothesize that the increase in fenestration area may facilitate the passage of large substances such as chylomicrons.

Much attention has been paid to diseases associated with a high-fat diet, represented by the Western diet (Cordner and Tamashiro 2015; Zinöcker and Lindseth 2018). Non-alcoholic fatty liver disease is considered to be caused by obesity and diabetes due to a high-fat diet (Fan et al. 2007; NeuschwanderTetri 2017). Severe steatosis and mild colitis are also important for the early occurrence of hepatocellular carcinoma (Sato et al. 2021). Thus, our findings may throw a new light for considering the mechanism of high fat-diet related diseases, as we consider that the dynamics of fenestration may be a regulation factor of nutrient absorption, especially lipid absorption.

Inflammatory bowel disease, especially Crohn's disease, is also associated with a high-fat diet (Owczarek et al. 2016). Although the pathophysiology of Crohn's disease remains unclear, abnormal immune reactions in the intestinal tract and high-fat diets could contribute to the development of Crohn's disease, and low-fat diets are recommended for treatment (Kakodkar and Mutlu 2017). Studies on dynamics of basal lamina fenestrations might throw a new light to find a clue to the solution of these problems, although further studies are needed to clarify this point.

In conclusion, the present study revealed that free cells passing through the fenestrations of the villous epithelial basal lamina increased in the rat jejunum $2 \mathrm{~h}$ after feeding, resulting in an increase in the pre-existing fenestration size in this region. This suggests that the enlargement of the fenestrations may increase the efficiency of lipid absorption by facilitating the movement of chylomicrons from the intracellular space to the lamina propria. Because basal lamina fenestrations are considered to play a role in fat absorption, as well as intestinal immunity, it might be interesting to study the dynamics of fenestrations in animal models of various diseases caused by a high-fat diet.

\section{Acknowledgements}

The authors thank the members of the Division of Microscopic Anatomy and Division of Gastroenterology and Hepatology, Niigata University Graduate School of Medical and Dental Sciences, Niigata, Japan, for their guidance and encouragement. We would like to thank Editage (www.editage.com) for English language editing.

\section{CONFLICT OF INTEREST}

This study was funded by Towa Pharmaceutical Co., Ltd. (MS, TU and ST)

\section{REFERENCES}

Azumi R, Morita K, Mizutani Y, Hayatsu M, Terai S et al. (2018) Dynamics of basal lamina fenestrations in the rat intestinal villous epithelium in response to dietary conditions. Biomed Res (Tokyo) 39, 65-74.

Chelakkot C, Ghim J and Ryu SH (2018) Mechanisms regulating intestinal barrier integrity and its pathological implications. Exp Mol Med 50, 1-9.

Cordner ZA and Tamashiro KL (2015) Effects of high-fat diet exposure on learning \& memory. Physiol Behav 152, 363-371.

Fan JG, Saibara T, Chitturi S, Kim BI, Sung J et al. (2007) What are the risk factors and settings for non-alcoholic fatty liver disease in Asia-Pacific? J Gastroenterol Hepatol 22, 794800.

Furuya K, Sokabe M and Furuya S (2005) Characteristics of subepithelial fibroblasts as a mechano-sensor in the intestine: cell-shape-dependent ATP release and P2Y1 signaling. J Cell Sci 118, 3289-3304.

Hara Y, Miura S, Komoto S, Inamura T, Koseki S et al. (2003) Exposure to fatty acids modulates interferon production by intraepithelial lymphocytes. Immunol Lett 86, 139-148.

Hoshi O and Ushiki T (2004) Neutrophil extravasation in rat mesenteric venules induced by the chemotactic peptide $\mathrm{N}$-formyl-methionyl-luecylphenylalanine (fMLP), with special attention to a barrier function of the vascular basal lamina for neutrophil migration. Arch Histol Cytol 67, 107-114.

Ji Y, Sakata Y and Tso P (2011) Nutrient-induced inflammation in the intestine. Curr Opin Clin Nutr Metab Care 14, 315321.

Kakodkar S and Mutlu EA (2017) Diet as a therapeutic option for adult inflammatory bowel disease. Gastroenterol Clin North Am 46, 745-767.

Kiela PR and Ghishan FK (2016) Physiology of intestinal absorption and secretion. Best Pract Res Clin Gastroenterol 30, 145-159.

Komuro T (1985) Fenestrations of the basal lamina of intestinal villi of the rat. Scanning and transmission electron microscopy. Cell Tissue Res 239, 183-188.

Kruskal WH and Wallis WA (1952) Use of ranks in one-criterion variance analysis. J Am Stat Assoc 47, 583-621.

Mantaj J, Abu-Shams T, Enlo-Scott Z, Swedrowska M and Vllasaliu D (2018) Role of the basement membrane as an intestinal barrier to absorption of macromolecules and nanoparticles. Mol Pharm 15, 5802-5808.

McClugage SG and Low FN (1984) Microdissection by ultrason- 
ication: porosity of the intestinal epithelial basal lamina. $\mathrm{Am}$ $J$ Anat 171, 207-216.

Miura S, Tsuzuki Y, Hokari R and Ishii H (1998) Modulation of intestinal immune system by dietary fat intake: relevance to Crohn's disease. J Gastroenterol Hepatol 13, 1183-1190.

Morita K, Azumi R, Sato M, Mizutani Y, Hayatsu M et al. (2019) Dynamic changes in basal lamina fenestrations in rat intestinal villous epithelium under high-fat diet condition. Biomed Res (Tokyo) 40, 57-66.

Neuschwander-Tetri BA (2017) Non-alcoholic fatty liver disease. BMC Med 15, 45.

Owczarek D, Rodacki T, Domagała-Rodacka R, Cibor D and Mach T (2016) Diet and nutritional factors in inflammatory bowel diseases. World J Gastroenterol 22, 895-905.

Sato T, Tsuchiya A, Owaki T, Kumagai M, Motegi S et al. (2021) Severe steatosis and mild colitis are important for the early occurrence of hepatocellular carcinoma. Biochem Biophys Res Commun 566, 36-44.
Takahashi-Iwanaga H, Iwanaga T and Isayama H (1999) Porosity of the epithelial basement membrane as an indicator of macrophage-enterocyte interaction in the intestinal mucosa. Arch Histol Cytol 62, 471-481.

Takeuchi T and Gonda T (2004) Distribution of the pores of epithelial basement membrane in the rat small intestine. $J$ Vet Med Sci 66, 695-700.

Tso P and Balint JA (1986) Formation and transport of chylomicrons by enterocytes to the lymphatics. Am J Physiol 250, G715-G726.

Walker DC, Behzad AR and Chu F (1995) Neutrophil migration through preexisting holes in the basal laminae of alveolar capillaries and epithelium during streptococcal pneumonia. Microvas Res 50, 397-416.

Zinöcker MK and Lindseth IA (2018) The Western diet-microbiome-host interaction and its role in metabolic disease. Nutrients 10, 365 . 